\title{
Effect of incorporated star-shaped four-armed stereo diblock poly(lactide) on the crystallization behavior of linear one-armed poly(L-lactide) or poly(D-lactide)
}

\author{
Hideto Tsuji, Ryota Ozawa and Nobutsugu Matsumura \\ Polymer Journal (2016) 48, 209-213; doi:10.1038/pj.2015.92; published online 14 October 2015
}

\section{INTRODUCTION}

The improvement of thermal stability of biobased biodegradable poly (L-lactide), that is, poly(L-lactic acid) (PLLA), is a crucial issue for achieving its widespread use as an alternative material to petro-derived polymers. ${ }^{1-4}$ A promising means of improving the thermal stability of poly(lactide), that is, poly(lactic acid) (PLA)-based biodegradable material, is to elevate its crystallinity by adding a nucleating or crystallization accelerating agent. Some nucleating or crystallization accelerating agents have been reported for PLLA. ${ }^{5}$ Among these crystallization accelerating agents, stereocomplex (SC) crystallites of PLLA and poly(D-lactide), that is, poly(D-lactic acid) (PDLA), are known to be highly effective and have been extensively studied. ${ }^{6-12}$ In addition, a short PDLA chain connected to a long PLLA chain is able to act as a nucleating or crystallization accelerating agent. ${ }^{13}$ A relatively short PDLA chain induces stereocomplex formation, which results in the accelerated homo-crystallization of a PLLA chain.

Among stereoblock PLAs, four-armed stereo diblock PLAs with equimolar L- and D-lactide units (4-LD; Figure 1) predominantly form SC crystallites without the formation of homo-crystallites. ${ }^{14}$ The formation of SC crystallites by stereoblock PLAs composed of equimolar L- and D-lactide units such as 4-LD does not necessarily require the aid of a PLLA matrix for SC formation, which is in contrast to PDLA added to PLLA, ${ }^{6-12}$ and the formed SC crystallites are expected to act as promising crystallization accelerating agents for PLLA or PDLA homo-crystallization. However, in the case of 4-LD, which is a multi-armed stereo diblock PLA, there are two types of configurational combinations for the shell of a star-shaped 4-LD and a linear one-armed PLA, that is, the PLA shell of a 4-LD has an opposite or an identical configuration compared with the configuration of a linear one-armed PLA matrix. Although such configurational disagreement and agreement (Figure 1) are expected to affect the accelerating effect of 4-LD on homo-crystallization of linear onearmed PLA, this issue has not yet been explored. For the crystallization of equimolar 4-LD/PLLA and 4-LD/PDLA blends during solvent evaporation, SC crystallites were reported to be the main crystalline species, although the crystallinity was lower than the crystallinities of the linear one-armed PLLA/PDLA and linear one-armed PLLA or PDLA/linear one-armed stereo diblock PLA. ${ }^{15}$ However, this study focused on the crystallization behavior of equimolar blends but not on the accelerating effect of a small amount of 4-LD on PLLA or PDLA homo-crystallization.

In the present study, a high-molecular-weight linear one-armed PLLA and PDLA as well as a star-shaped four-armed stereo diblock PLA with a PDLA shell and PLLA core (4-LD) (Figure 1) were synthesized. In addition, a small amount of 4-LD (5 wt\%) was added to a linear one-armed PLLA or PDLA, and the acceleration of a linear one-armed PLLA or PDLA homo-crystallization by the incorporated 4-LD as a stereoblock PLA and the aforementioned effects of configurational disagreement and agreement were investigated.

\section{EXPERIMENTAL PROCEDURE}

\section{Materials}

High-molecular-weight PLLA (number-average molecular weight $M_{\mathrm{n}}=5.1 \times 10^{5} \mathrm{~g} \mathrm{~mol}^{-1}$, weight-average molecular weight $M_{\mathrm{w}} / M_{\mathrm{n}}=1.8$, specific optical rotation $\left([\alpha]_{589}^{25}\right)$ in chloroform (guaranteed grade, Nacalai Tesque,

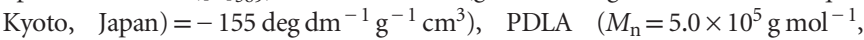
$M_{\mathrm{w}} / M_{\mathrm{n}}=1.7, \quad[\alpha]_{589}^{25}$ in chloroform $\left.=157 \mathrm{deg} \mathrm{dm}^{-1} \mathrm{~g}^{-1} \mathrm{~cm}^{3}\right)$ and 4-LD $\left(M_{\mathrm{n}}=5.5 \times 10^{4} \mathrm{~g} \mathrm{~mol}^{-1}, \quad M_{\mathrm{w}} / M_{\mathrm{n}}=1.1, \quad[\alpha]_{589}^{25}\right.$ in a mixed solvent of chloroform/1,1,1,3,3,3-hexafluoro-2-propanol (abbreviated as HPIP, HPLC grade, Nacalai Tesque; $\mathrm{vol} / \mathrm{vol}=95 / 5)=-4.5 \mathrm{deg} \mathrm{dm}^{-1} \mathrm{~g}^{-1} \mathrm{~cm}^{3}$, L-lactyl unit content $=51.4 \%$, averaged block lengths of PLLA and PDLA $=98$ and 93 monomer units, respectively) were synthesized and purified according to previously reported procedures. ${ }^{14,16}$ Neat PLLA and PDLA samples and samples with 5 wt $\%$ 4-LD added (abbreviated as PLLA/4-LD and PDLA/4-LD, respectively, thickness of $25 \mu \mathrm{m}$ ) were prepared by solution casting. Briefly, each solution of polymers was prepared with polymer concentration of $1.0 \mathrm{~g} \mathrm{dl}^{-1}$ using a mixed solvent consisting of dichloromethane/HFIP ( $\mathrm{vol} / \mathrm{vol}$ 95/5), and this solution was cast onto a petri dish and subjected to solvent evaporation at $25^{\circ} \mathrm{C}$ for approximately 1 day and then dried in vacuo for 6 days. For preparation of the blend samples (PLLA or PDLA with 4-LD), separately prepared solutions of two polymers were mixed vigorously before solvent evaporation. For preparation of the melt-quenched samples, each 


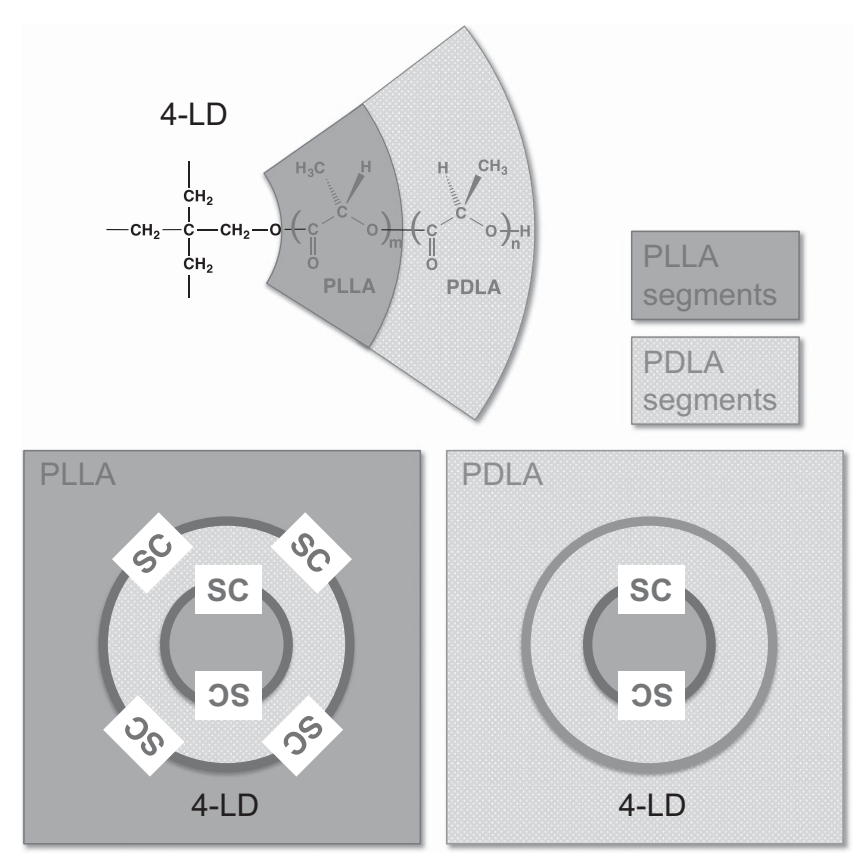

Figure 1 Molecular structure of 4-LD and material models of PLLA or PDLA with 4-LD. The gray and white parts are composed of PLLA and PDLA segments, respectively. 4-LD, four-armed stereo diblock poly(lactic acid) with equimolar L- and D-lactide units; PDLA, poly(D-lactic acid); PLLA, poly(L-lactic acid). A full color version of this figure is available at Polymer Journal online.

solution-cast sample packed in a DSC aluminum pan was sealed in a test tube under reduced pressure, melted at $190^{\circ} \mathrm{C}$ for $3 \mathrm{~min}$ and quenched at $0{ }^{\circ} \mathrm{C}$ for at least $5 \mathrm{~min}$.

\section{Physical measurements and observation}

The glass transition, cold crystallization, melting temperatures $\left(T_{\mathrm{g}}, T_{\mathrm{cc}}\right.$ and $T_{\mathrm{m}}$, respectively) and enthalpies of cold crystallization and melting $\left(\Delta H_{\mathrm{cc}}\right.$ and $\Delta H_{\mathrm{m}}$, respectively) of samples for heating and crystallization temperature and enthalpy ( $T_{\mathfrak{c}}$ and $\Delta H_{\mathfrak{c}}$, respectively) for slow cooling from the melt were determined with a Shimadzu (Kyoto, Japan) DSC-50 differential scanning calorimeter under nitrogen gas flow at a rate of $50 \mathrm{ml} \mathrm{min}^{-1}$. To investigate crystallization during heating, $\sim 3 \mathrm{mg}$ of melt-quenched samples were heated at a rate of $10^{\circ} \mathrm{C} \mathrm{min}^{-1}$ from ambient temperature to $230^{\circ} \mathrm{C}$. In addition, to investigate crystallization during slow cooling from the melt, $\sim 3 \mathrm{mg}$ of as-cast samples were heated at a rate of $10^{\circ} \mathrm{C} \mathrm{min}^{-1}$ from ambient temperature to $190^{\circ} \mathrm{C}$, held at this temperature for $1 \mathrm{~min}$ and then cooled at a rate of $3^{\circ}$ $\mathrm{C} \min ^{-1}$ to ambient temperature (crystallization was observed here). The $T_{\mathrm{g}}$, $T_{\mathrm{cc}}, T_{\mathrm{m}}, \Delta H_{\mathrm{cc}}$ and $\Delta H_{\mathrm{m}}$ values were calibrated using tin, indium and benzophenone as standards. By definition, $\Delta H_{\mathrm{cc}}$ and $\Delta H_{\mathrm{m}}$ are negative and positive, respectively. The crystalline species and crystallinity $\left(X_{\mathrm{c}}\right)$ values of the samples were estimated using wide-angle X-ray diffractometry (WAXD). The WAXD measurements were performed at $25^{\circ} \mathrm{C}$ using a RINT-2500 (Rigaku, Tokyo, Japan) equipped with a $\mathrm{Cu}-\mathrm{K} \alpha$ source $(\lambda=1.5418 \AA)$, which was operated at $40 \mathrm{kV}$ and $200 \mathrm{~mA}$. To exclude issues related to the probable heterogeneity of the samples, we performed measurements with triplicate samples from different positions on the solution-cast samples, and the physical properties were averaged values for the triplicate samples. The relatively high reproducibility of the $T_{\mathrm{cc}}, T_{\mathrm{c}}, \Delta H_{\mathrm{cc}} \Delta H_{\mathrm{c}}$ and $X_{\mathrm{c}}$ values strongly suggested that the heterogeneity was very small and did not affect our conclusions.

\section{RESULTS}

\section{Differential scanning calorimetry}

The crystallization of the melt-quenched samples for heating and the as-cast samples for slow cooling from the melt was monitored by DSC (Figures $2 \mathrm{a}$ and $\mathrm{b}$ ), and the entire DSC thermograms for heating in a wider temperature range are shown in Supplementary Figure S1. The thermal properties were estimated from the DSC thermograms in Figures $2 \mathrm{a}$ and $\mathrm{b}$. Thus, the obtained $T_{\mathrm{cc}}, \Delta H_{\mathrm{m}}(\mathrm{H}+\mathrm{S})$ and $T_{\mathrm{c}}$ are plotted in Figures $2 \mathrm{c}$ and d, and the other thermal properties are listed in Supplementary Table S1. For heating, the neat PLLA and PDLA had $T_{\mathrm{cc}}$ values of 116.3 and $111.9^{\circ} \mathrm{C}$, respectively, whereas the $T_{\mathrm{cc}}$ values of PLLA/4-LD and PDLA/4-LD (104.0 and $106.8^{\circ} \mathrm{C}$, respectively) were correspondingly much lower and slightly lower than the values of the neat PLLA and PDLA (Figure 2c). For slow cooling, the neat PLLA and PDLA had $T_{\mathrm{c}}$ values of 105.8 and $108.3^{\circ} \mathrm{C}$, respectively, whereas the $T_{\mathrm{c}}$ values of PLLA/4-LD and PDLA/4-LD (123.3 and $108.3^{\circ} \mathrm{C}$, respectively) were much higher than and in agreement with the values of the neat PLLA and PDLA, respectively (Figure 2d). These findings indicated that the incorporated 4-LD facilitated and accelerated PLLA homo-crystallization during heating and slow cooling and PDLA homo-crystallization during heating and that the accelerating effect of 4-LD was much higher for the linear one-armed PLLA than for the linear one-armed PDLA.

$\Delta H_{\mathrm{m}}(\mathrm{H}+\mathrm{S})=\Delta H_{\mathrm{m}}(\mathrm{H})+\Delta H_{\mathrm{m}}(\mathrm{S})$, which is an indicator of maximum crystallinity during heating, is plotted in Figure 2c. As shown in Figure 2c and Supplementary Table S1, the $\Delta H_{\mathrm{m}}(\mathrm{H}+\mathrm{S})$ and $\Delta H_{\mathrm{m}}(\mathrm{H})$ of all samples were similar to one another, although the $\Delta H_{\mathrm{m}}(\mathrm{H})$ values of PLLA/4-LD and PDLA/4-LD were slightly lower than the values of the neat PLLA and PDLA owing to the formation of SC crystallites as evidenced by the non-zero $\Delta H_{\mathrm{m}}(\mathrm{S})$ values of PLLA/4-LD and PDLA/4-LD (Supplementary Table S1), which should have reduced the amount of homo-crystallizable PLLA or PDLA chains in PLLA/4-LD and PDLA/4-LD.

\section{Wide-angle X-ray diffractometry}

To investigate the crystalline species and $X_{c}$ of the samples after meltquenching and slow cooling from the melt as in the DSC measurements, WAXD measurements were performed. The structures of the former samples should be identical to those samples before heating the melt-quenched samples or slow cooling from the melt of the as-cast samples. For the melt-quenched samples (Figure 3a), the neat PLLA and PDLA showed broad diffractions specific to amorphous materials, whereas PLLA/4-LD and PDLA/4-LD had crystalline diffractions at $2 \theta=12,21$ and $24^{\circ}$ specific to SC crystallites, ${ }^{16,17}$ which are indicated by arrows in Figure 3. This result indicates that the SC crystallites were contained in the melt-quenched PLLA/4-LD and PDLA/4-LD before DSC heating and as-cast PLLA/4-LD and PDLA/4-LD before slow cooling from the melt. The formed SC crystallites acted as a nucleating agent for linear one-armed PLLA or PDLA. All slowly cooled samples from the melt showed crystalline diffractions at $2 \theta=15,17,19$ and $22.5^{\circ}$, which corresponded to the $\alpha$-form of PLLA or PDLA homocrystallites, ${ }^{18,19}$ and the diffraction peaks due to the SC crystallites in PLLA/4-LD and PDLA/4-LD remained (Figure 3b). These findings were indicative of the fact that the incorporated 4-LD and SC crystallites did not affect the crystalline modification of the PLLA or PDLA homo-crystallites.

The $X_{\mathrm{c}}$ values of the homo- and SC crystallites $\left[X_{\mathrm{c}}(\mathrm{H})\right.$ and $X_{\mathrm{c}}(\mathrm{S})$ values, respectively] were estimated from the WAXD profiles in Figure 3. The obtained $X_{\mathrm{c}}(\mathrm{H})$ and $X_{\mathrm{c}}(\mathrm{S})$ values are summarized in Supplementary Table S2, and the total crystallinity $\left(X_{c}(\mathrm{H}+\mathrm{S})=X_{c}(\mathrm{H})\right.$ $\left.+X_{c}(S)\right)$ is plotted in Figure 2d. As shown in Supplementary Table S2, the $X_{\mathrm{c}}(\mathrm{S})$ value of the melt-quenched PLLA/4-LD (6.6\%) was higher than the corresponding value of the melt-quenched PDLA/ 4-LD (4.1\%). This result indicates that the amount of SC crystallites as a crystallization accelerating agent was affected by configurational disagreement and agreement and was higher for PLLA/4-LD (opposite 
a

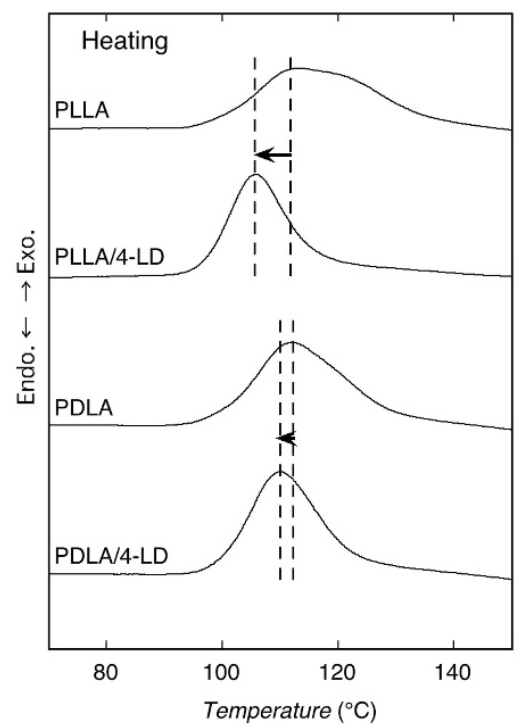

C

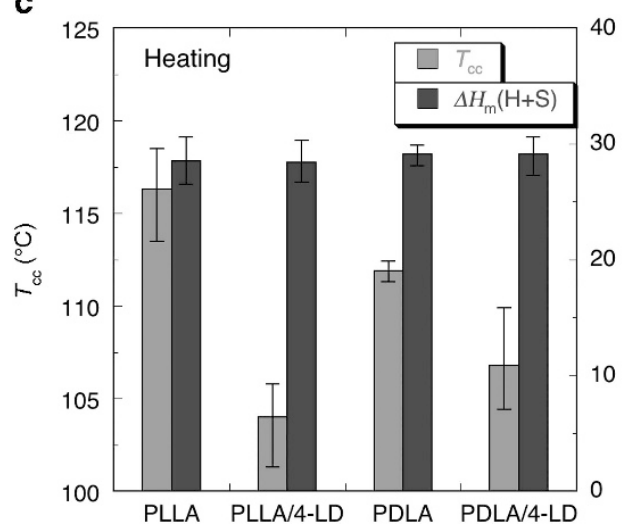

b

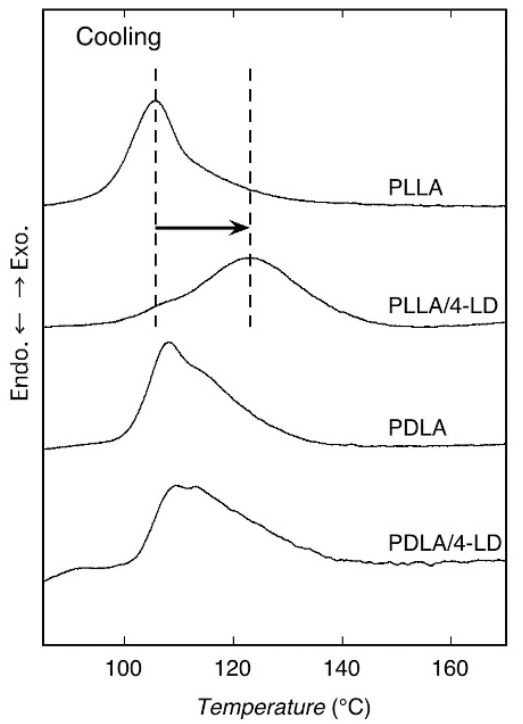

d

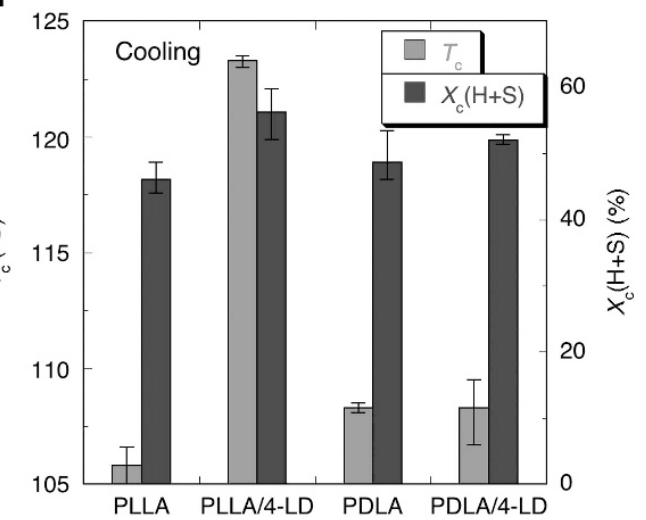

Figure 2 DSC thermograms of melt-quenched samples during heating (a) and as-cast samples during slow cooling from the melt $(\mathbf{b}), T_{\mathrm{cc}}$ and $\Delta H_{\mathrm{m}}(\mathrm{H}+\mathrm{S})$ of melt-quenched samples during heating (c) and $T_{c}$ and $X_{c}$ of as-cast samples during cooling from the melt (d). DSC, differential scanning calorimetry; 4-LD, four-armed stereo diblock poly(lactic acid) with equimolar L- and D-lactide units; PDLA, poly(D-lactic acid); PLLA, poly(L-lactic acid). A full color version of this figure is available at Polymer Journal online.
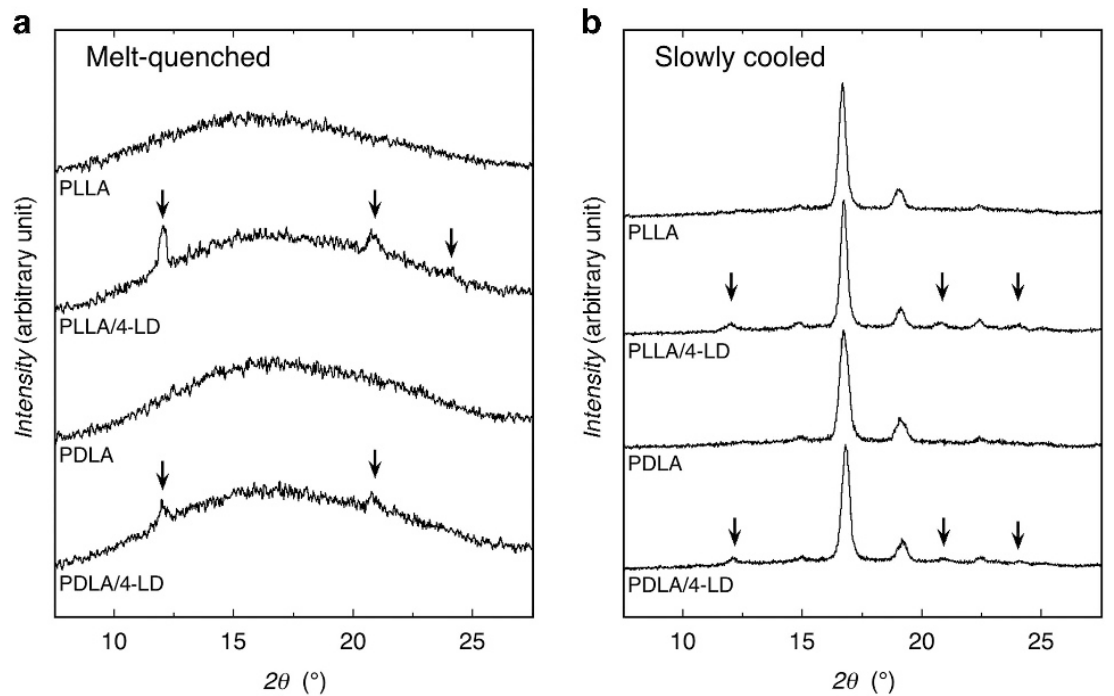

Figure 3 WAXD profiles of melt-quenched samples (a) and as-cast samples after slow cooling from the melt (b). 4-LD, four-armed stereo diblock poly(lactic acid) with equimolar L- and D-lactide units; PDLA, poly(D-lactic acid); PLLA, poly(L-lactic acid); WAXD, wide-angle X-ray diffractometry. 
configurations) than for PDLA/4-LD (identical configurations). After crystallization during slow cooling, the total crystallinity $\left(X_{\mathrm{c}}(\mathrm{H}+\mathrm{S})\right)$ of PLLA/4-LD (56.2\%) was $20 \%$ higher than that of the neat PLLA $(46.1 \%)$, whereas the $X_{\mathrm{c}}(\mathrm{H}+\mathrm{S})$ of PDLA/4-LD $(52.0 \%)$ was similar to that of the neat PDLA (48.7\%).

\section{Polarized optical microscopy}

To further investigate the effects of the SC crystallites and configurational disagreement or agreement on PLLA or PDLA homo-crystallization, a POM observation was performed. Figure 4 shows the polarized optical photomicrographs of the samples crystallized during slow cooling from the melt. In the neat PLLA and PDLA, homocrystallization started at $140{ }^{\circ} \mathrm{C}$ and $20-30 \%$ and most of the samples were covered by spherulites at 120 and $100^{\circ} \mathrm{C}$, respectively. For PDLA/ 4-LD, crystallization behavior similar to that of the neat PLLA and PDLA was observed, although the number of spherulites per unit area was lower for PDLA/4-LD than for the neat PLLA and PDLA. It is probable that although a portion of 4-LD formed SC crystallites and functioned as a nucleating agent, the remaining 4-LD acted as an impurity, which was similar to the case of the linear one-armed PLLA added to a low concentration of linear one-armed PDLA (for example, $0.1 \%) .{ }^{10}$ In PLLA/4-LD, the highest numbers of spherulites per unit area were formed and occupied $\sim 20 \%$ of the sample at $140{ }^{\circ} \mathrm{C}, \sim 80 \%$ at $120^{\circ} \mathrm{C}$ and the entire sample at $100^{\circ} \mathrm{C}$. The area covered with spherulites in PLLA $/ 4-\mathrm{LD}$ at $120^{\circ} \mathrm{C}$ was much larger than the area covered in the neat PLLA, PDLA and PDLA/4-LD, indicating accelerated crystallization of PLLA/4-LD during slow cooling.

\section{DISCUSSION}

Among the biobased biodegradable stereoblock PLAs, star-shaped four-armed stereo diblock PLA with equimolar L- and D-lactide units, $4-\mathrm{LD}$, was first revealed to accelerate the crystallization of linear onearmed PLLA during heating and slow cooling and linear one-armed PDLA during heating; the accelerating effect of 4-LD was much higher for linear one-armed PLLA than for linear one-armed PDLA. The accelerated homo-crystallization in PLLA/4-LD and PDLA/4-LD compared with the neat one-armed PLLA and one-armed PDLA was attributable to the presence of SC crystallites, which remained unmelted during the melting of the homo-crystallites and can act as crystallization accelerating agents during heating and slow cooling. In addition, the opposite configurations of linear one-armed PLA and the shell of 4-LD in PLLA/4-LD enhanced the accelerating effect of 4 - $\mathrm{LD}$, whereas the accelerating effect of 4 -LD was weaker owing to the identical configurations of the one-armed PLA and the shell of 4-LD, as in PDLA/4-LD.

This difference can be partly attributed to differences in the numbers of SC crystallites as revealed by the WAXD measurements (Supplementary Table S2), that is, the larger numbers of SC crystallites in PLLA/4-LD should have a stronger accelerating effect than the SC crystallites in PDLA/4-LD. The differences in the numbers of SC
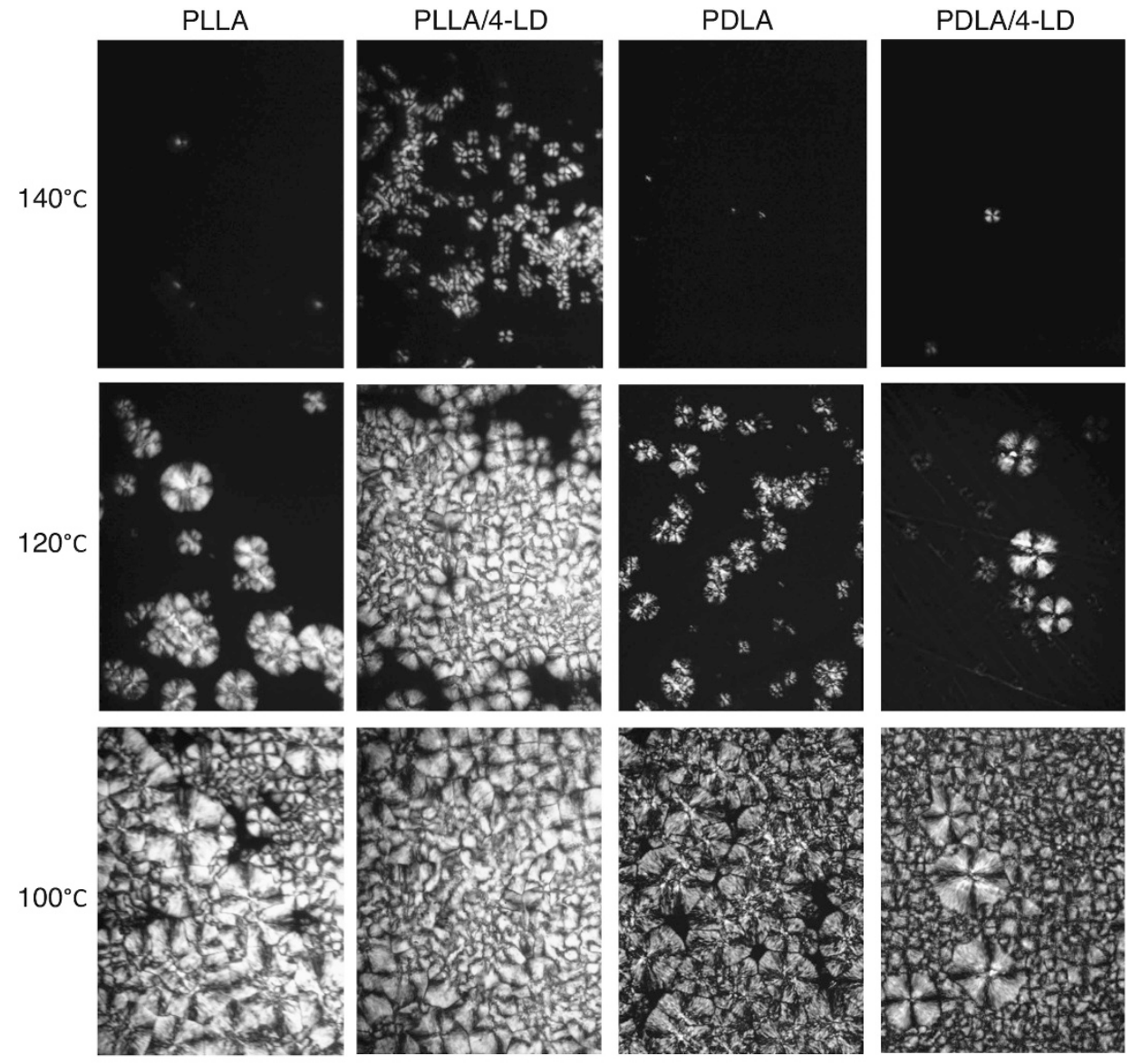

$100 \mu \mathrm{m}$

Figure 4 Polarized photomicrographs of samples obtained at the indicated temperatures during slow cooling from the melt. 4-LD, four-armed stereo diblock poly(lactic acid) with equimolar L- and D-lactide units; PDLA, poly(D-lactic acid); PLLA, poly(L-lactic acid). 
crystallites can be explained as follows. As shown in Figure 1, in the case of PLLA/4-LD with opposite configurations between the onearmed PLLA and the PDLA shell of 4-LD, the PDLA shell of 4-LD can form SC crystallites with the PLLA core of 4-LD, as well as the onearmed PLLA surrounding 4-LD, whereas in the case of PDLA/4-LD with identical configurations between the one-armed PDLA and the PDLA shell of 4-LD, the PDLA shell of 4-LD can form SC crystallites with the PLLA core of 4-LD. However, we must consider the possibility that the one-armed PDLA can penetrate into the core of 4-LD and form SC crystallites with the PLLA core. Moreover, in PLLA/4-LD, the formation of SC crystallites between the one-armed PLLA and the PDLA shell of 4-LD should increase the interaction between the SC crystallites and the one-armed PLLA matrix, resulting in increased numbers of spherulite nuclei and highly accelerated homo-crystallization. However, in PDLA/4-LD, strong interactions between the one-armed PDLA and the SC crystallites cannot be expected because the probability of the one-armed PDLA matrix to take part in SC formation is very low, and the SC crystallites formed inside 4-LD were confined. This type of interfacial interaction between SC crystallites and one-armed PLLA has been previously suggested to affect the nucleation of SC crystallites. ${ }^{12}$ In addition, it is possible that the PLLA and PDLA chains of 4-LD may form SC crystallites and that the remaining PDLA chains of $4-\mathrm{LD}$, which did not take part in SC formation, must be rather short. The SC crystallites covered with these short PDLA chains, which would form homo-crystallites with a low melting point, may not act as effective crystallization accelerating agents for PDLA. Another probable explanation is as follows. The stability of the SC crystallites formed between the one-armed PLLA and the PDLA shell of 4-LD in PLLA/4-LD during solvent evaporation and the 1st heating was greater than the stability of the SC crystallites formed between the PDLA shell of 4-LD or the one-armed PDLA and PLLA core of 4-LD in PDLA/4-LD, resulting in greater crystallization accelerating ability.

The incorporated 4-LD significantly increased $X_{\mathrm{c}}$ of PLLA/4-LD after slow cooling from the melt, which should be useful for the preparation of PLLA-based materials with high crystallinities. In addition, the $\Delta H_{\mathrm{m}}(\mathrm{H}+\mathrm{S})$ of PLLA/4-LD and PDLA/4-LD during heating and $X_{\mathrm{c}}(\mathrm{H}+\mathrm{S})$ of PDLA/4-LD after slow cooling were similar to enthalpies of the neat PLLA and PDLA. However, the $\Delta H_{\mathrm{m}}(\mathrm{H}+\mathrm{S})$ of PLLA/4-LD during heating and $X_{c}(\mathrm{H}+\mathrm{S})$ of PLLA/4-LD are expected to be higher compared with the enthalpies of neat PLLA when the heating or cooling rate is elevated.

\section{CONCLUSIONS}

The incorporated 4-LD formed SC crystallites and facilitated and accelerated the homo-crystallization of linear one-armed PLLA during heating and slow cooling and linear one-armed PDLA during heating. The accelerating effect was greater for PLLA/4-LD with the opposite configurations of linear one-armed PLLA and the PDLA shell of 4-LD than for PDLA/4-LD with identical configurations in linear one-armed PDLA and the PDLA shell of 4-LD. In addition to the finding that the opposite configurations in PLLA/4-LD increased the numbers of SC crystallites, the expected stronger interaction between one-armed PLLA and the SC crystallites should have increased the number of spherulite nuclei per unit area and the accelerating effect of 4-LD on the homo-crystallization of one-armed PLA compared with PDLA/ 4-LD. Furthermore, the incorporation of 4-LD or SC crystallites effectively increased the $X_{c}$ of PLLA/4-LD for slow cooling but did not affect the crystalline modifications of PLLA or PDLA homo-crystallites in PLLA/4-LD and PDLA/4-LD for slow cooling.

\section{CONFLICT OF INTEREST}

The authors declare no conflict of interest.

\section{ACKNOWLEDGEMENTS}

This research was supported by JSPS KAKENHI Grant Number 24550251 and MEXT KAKENHI Grant Number 24108005.

1 Kharas, G. B., Sanchez-Riera, F. \& Severson, D. K. in Plastics from Microbes (ed. Mobley, D. P.) Ch. 4, 93-137 (Hanser Publishers, New York, NY, USA, 1994).

2 Hartmann, M. H. in Biopolymers from Renewable Resources (ed. Kaplan, D. L.) Ch. 15, 367-411 (Springer, Berlin, Germany, 1998).

3 Auras, R., Lim, L.-T., Selke, S. E. M. \& Tsuji, H. in Poly(lactic acid): Synthesis, Structures, Properties, Processing, and Applications (Wiley Series on Polymer Engineering and Technology), (John Wiley \& Sons, NJ, USA, 2010).

4 Pang, X., Zhuang, X., Tang, Z. \& Chen, X. Polylactic acid (PLA): Research, development and industrialization. Biotech. J. 5, 1125-1136 (2010).

5 Tsuji, H. in Bio-Based Plastics: Materials and Applications (Wiley Series in Renewable Resource), (eds Kabasci, S., Series Stevens, C.) Ch. 8, 171-239 (John Wiley \& Sons, West Sussex, UK, 2014).

6 Brochu, S., Prud'homme, R. E., Barakat, I. \& Jérôme, R. Stereocomplexation and morphology of polylactides. Macromolecules 28, 5230-5239 (1995).

7 Schmidt, S. C. \& Hillmyer, M. A. Polylactide stereocomplex crystallites as nucleating agents for isotactic polylactide. J. Polym. Sci. B Polym. Phys. 39, 300-313 (2001).

8 Yamane, H. \& Sasai, K. Effect of the addition of poly(D-lactic acid) on the thermal property of poly(L-lactic acid). Polymer 44, 2569-2575 (2003).

9 Anderson, K. S. \& Hillmyer, M. A. Melt preparation and nucleation efficiency of polylactide stereocomplex crystallites. Polymer 47, 2030-2035 (2006).

10 Tsuji, H., Takai, H. \& Saha, S. K. Isothermal and non-isothermal crystallization behavior of poly (L-lactic acid): effects of stereocomplex as nucleating agent. Polymer 47, 3826-3837 (2006).

11 Rahman, N., Kawai, T., Matsuba, G., Nishida, K., Kanaya, T., Watanabe, H., Okamoto, H., Kato, M., Usuki, A., Matsuda, M., Nakajima, K. \& Honma, N. Effect of polylactide stereocomplex on the crystallization behavior of poly(L-lactic acid). Macromolecules 42, 4739-4745 (2009).

12 Narita, J., Katagiri, M. \& Tsuji, H. Highly enhanced accelerating effect of meltrecrystallized stereocomplex crystallites on poly (L-lactic acid) crystallization, 2-effects of poly(D-lactic acid) concentration. Maromol. Mater. Eng. 298, 270-282 (2013).

13 Tsuji, H. \& Tajima, T. Crystallization behavior of stereo diblock poly(lactide)s with relatively short poly(D-Lactide) segment from partially melted state. Macromol. Mater. Eng. 299, 1089-1105 (2014).

14 Tsuji, H. \& Yamashita, Y. Highly accelerated stereocomplex crystallization by blending star-shaped 4-armed stereo diblock poly(lactide)s with poly(D-lactide) and poly(L-lactide) cores. Polymer 55, 6444-6450 (2014).

15 Shao, J., Tang, Z., Sun, J., Li, G. \& Chen, X. Linear and four-armed poly(l-lactide)block-poly(d-lactide) copolymers and their stereocomplexation with poly(lactide)s. J. Polym. Sci. B Polym. Phys. 52, 1560-1567 (2014).

16 Tsuji, H. \& Ikada, Y. Stereocomplex formation between enantiomeric poly (lactic acid) s. XI. Mechanical properties and morphology of solution-cast films. Polymer 40, 6699-6708 (1999).

17 Tsuji, H. Poly(lactide) stereocomplexes: formation, structure, properties, degradation, and applications. Macromol. Biosci. 5, 569-597 (2005).

18 Pan, P., Kai, W., Zhu, B., Dong, T. \& Inoue, Y. Polymorphous crystallization and multiple melting behavior of poly(L-lactide): molecular weight dependence. Macromolecules 40, 6898-6905 (2007).

19 Kawai, T., Rahman, N., Matsuba, G., Nishida, K., Kanaya, T., Nakano, M., Okamoto, H., Kawada, J., Usuki, A., Honma, N., Nakajima, K. \& Matsuda, M. Crystallization and melting behavior of poly(L-lactic acid). Macromolecules 40, 9463-9469 (2007).

Supplementary Information accompanies the paper on Polymer Journal website (http://www.nature.com/pj) 